\title{
GPER mediated estrogenic amelioration of sodium channel dysfunction in stressed human induced pluripotent stem cell-derived cardiomyocytes
}

Xide $\mathrm{Hu}^{1}$

${ }^{1}$ Affiliation not available

November 8, 2021

\section{Running Title}

Estrogen alters cardiac sodium currents through GPER

\section{Author names}

Xide $\mathrm{Hu}^{1}$, Lu Fu${ }^{1}$, Mingming Zhao ${ }^{1}$, Hongyuan Zhang ${ }^{1,2}$, Zheng Gong ${ }^{3}$, Tongtong Ma ${ }^{1}$, Jeremiah Ong'achwa Machuki ${ }^{1}$, Gabriel Komla Adzika ${ }^{1}$, Xiaomei Liu ${ }^{4}$, Renxian Tang ${ }^{4}$, Hong Sun ${ }^{*}$

\section{Affiliations}

${ }^{1}$ Physiology Department, Xuzhou Medical University, Xuzhou, China.

2 Division of Cardiovascular Science, School of medicine, The University of Manchester, Manchester, UK.

3 School of Public Affairs \& Governance, Silliman University, Philippines.

${ }^{4}$ Department of Pathogenic Biology and Immunology, Xuzhou Medical University, Xuzhou, China.

\section{* Corresponding author}

Prof. Hong Sun, MD, PhD

Physiology Department, Xuzhou Medical University, 209 Tongshan Road, Xuzhou, Jiangsu, 221004, China

E-mail: sunh@xzhmu.edu.cn

\section{Supplemental material \\ Supplementary figures / tables}


A

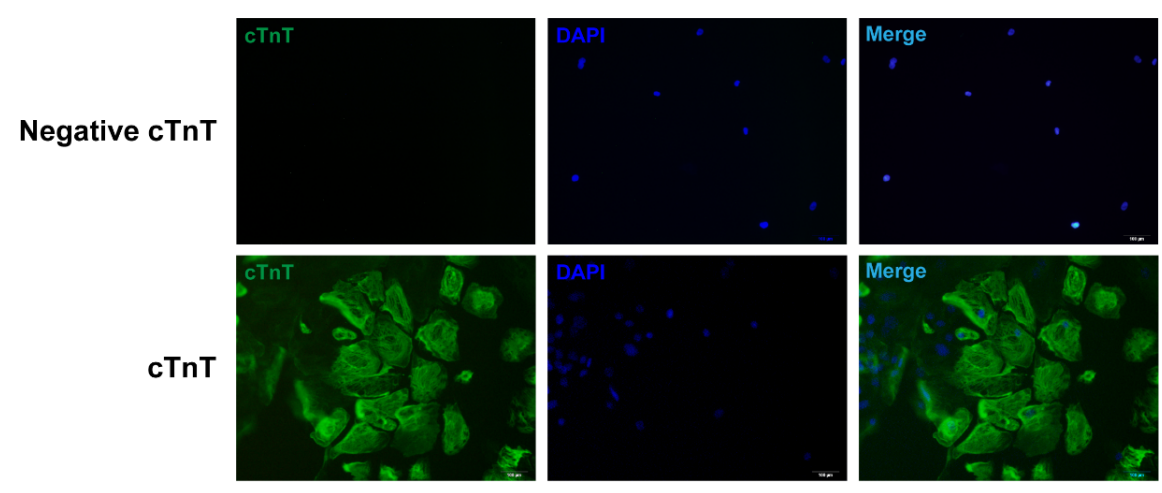

B

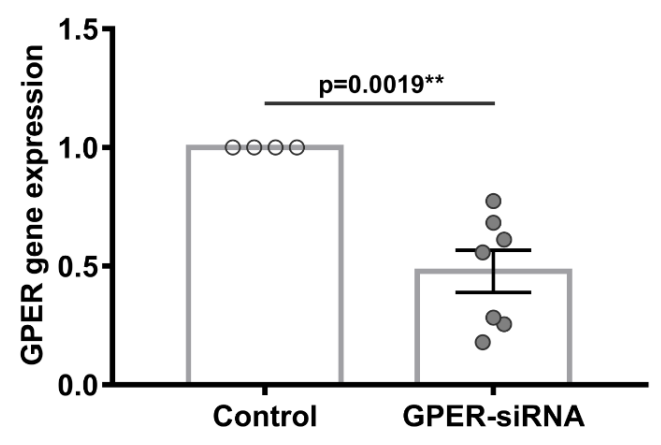

Supplemental Figure 1 Abundant expressions of cardiac troponin T and decreasing expressions of GPER gene after siRNA interference in hiPSC-CMs. (A) immunofluorescence of cTnT (green) and DAPI (blue). $\mathrm{cTnT}$, cardiac troponin T; Bars $=100 \mu \mathrm{m}$. (B) Relative expression of GPER gene after siRNA knockdown. Unpaired t test was used. ${ }^{* *} P<0.01$. Data are shown as Mean \pm S.E.M. 


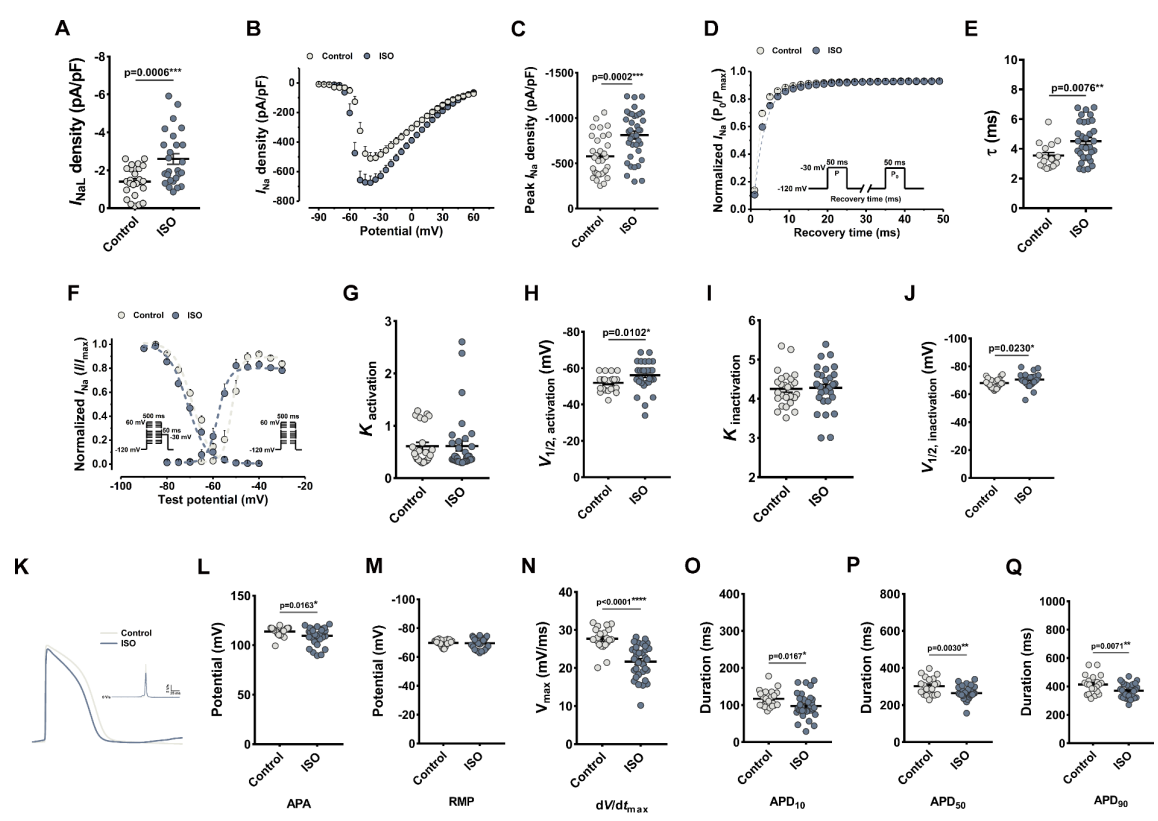

Supplemental Figure 2 ISO elevates sodium currents and alteres $I \mathrm{Na}$ kinetics as well as action potential parameters in hiPSC-CMs. (A) Average $I$ NaLdensity. (B) Current-voltage curve of the sodium channel. (C) Peak $I \mathrm{Na}$ density. (D) Time constant of recovery from inactivation curve of the sodium channel. (E) $\tau$ value of recovery from inactivation. (F) Activation and inactivation curve of the sodium channel of hiPSC-CMs. (G-J) Values for $k$ and $\mathrm{V}_{1 / 2}$ of (in) activation. $\mathrm{n}=17-37$ cells, from 9-11 petri dishes. (K) Representative single $\mathrm{AP}$ and slope curve of hiPSC-CMs. (L-Q) Values for APA, RMP, d $V / \mathrm{d} t$ max $, \mathrm{APD}_{10}, \mathrm{APD}_{50}$ and $\mathrm{APD}_{90}$. $\mathrm{n}=20-34$ cells, from 4 petri dishes, separately. An unpaired t test was used. Welch's correction was selectively used in cases of uneven variance. ${ }^{*} p<0.05,{ }^{* *} p<0.01,{ }^{* * *} p<0.001,{ }^{* * * *} p<0.0001$. ISO, isoproterenol; $I_{\mathrm{NaL}}$, late sodium current; $I_{\mathrm{Na}}$, sodium current; $k$ of (in) activation, slope factor of (in) activation; $\mathrm{V}_{1 / 2}$ of (in) activation, half-voltage of (in) activation; $\tau$, time constant of recovery from inactivation; APA, action potential amplitude; RMP, resting membrane potential; $\mathrm{d} V / \mathrm{d} t \max$, maximal action potential upstroke velocity; $\mathrm{APD}_{10}, 50,90$, action potential duration at $10 \%, 50 \%$ and $90 \%$ repolarization. pA, pico Ampere; pF, pico Farad. Insets: voltage clamp protocols. 

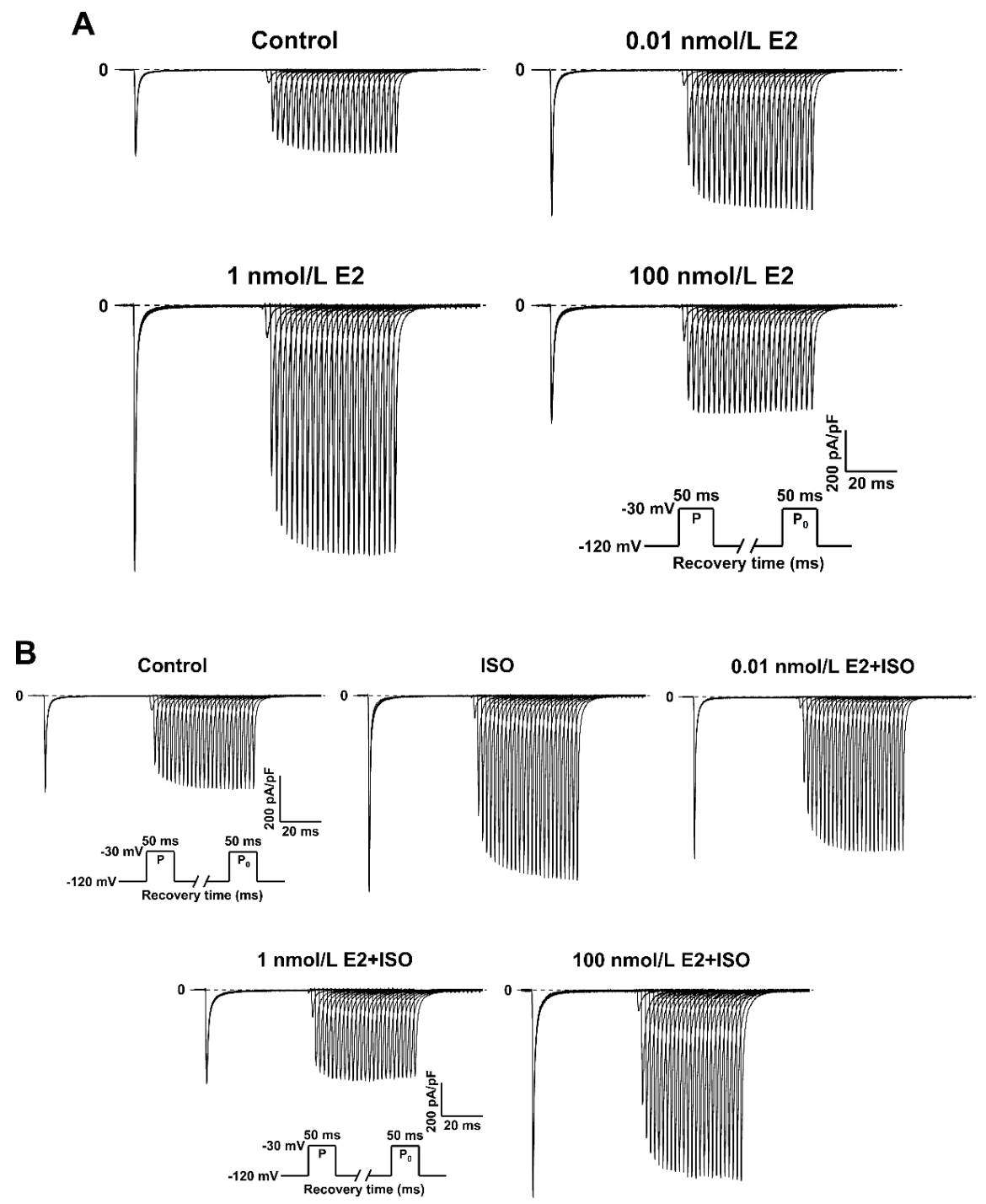

Supplemental Figure 3 Effects of estrogen on the time constant of recovery from inactivation of sodium current in hiPSC-CMs. (A and B) Representative traces were showed in each group. Insets: voltage clamp protocols. 
A

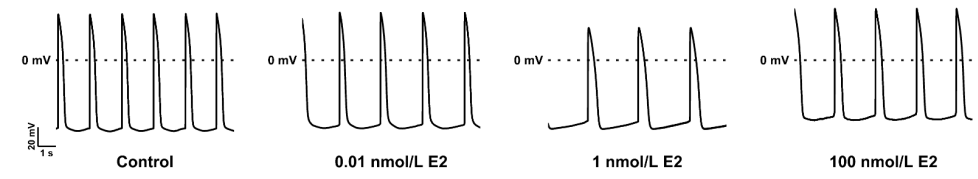

B

C

D

E
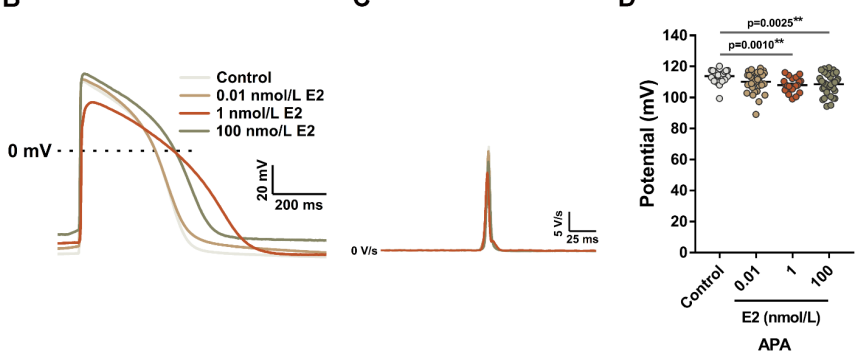

$\mathbf{F}$

G
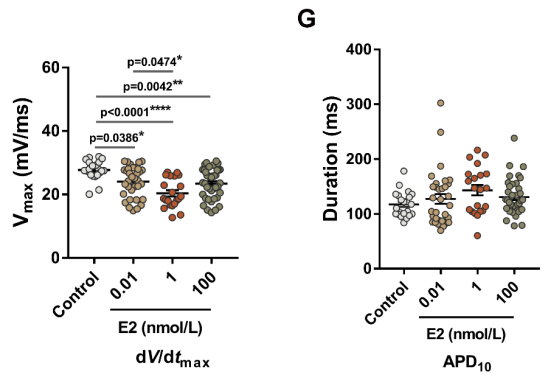

H

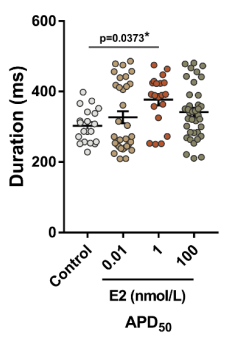

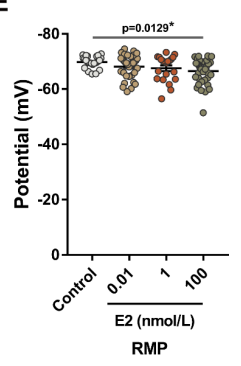

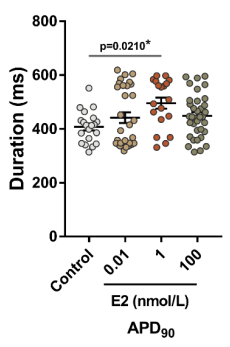

Supplemental Figure 4 E2 modifies the action potential parameters of hiPSC-CMs. (A-C) Typical APs and slope curve of hiPSC-CMs. (D-I) Values for APA, RMP, $\mathrm{d} V / \mathrm{d} t$ max $, \mathrm{APD}_{10}, \mathrm{APD}_{50}$ and $\mathrm{APD}_{90} . \mathrm{n}=19-40$ cells, from $4-6$ petri dishes. ${ }^{*} p<0.05,{ }^{* *} p<0.01,{ }^{* * * *} p<0.0001$. 


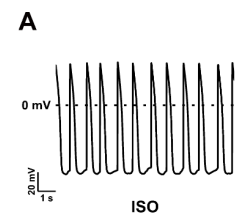

ISO

B

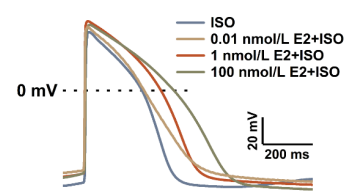

F

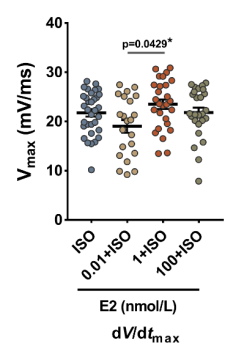

G

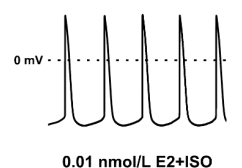

c
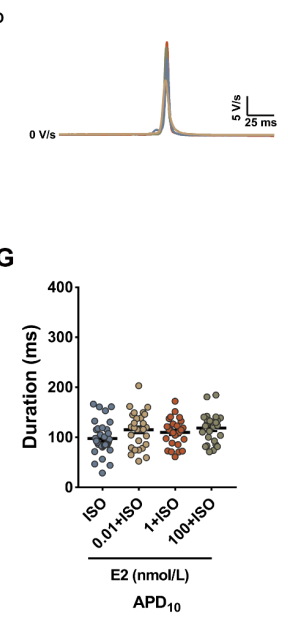

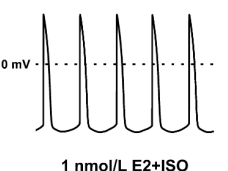

D

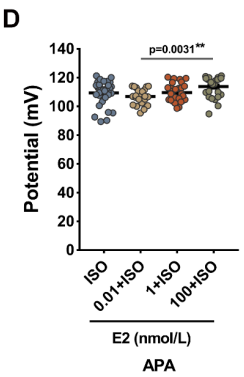

H

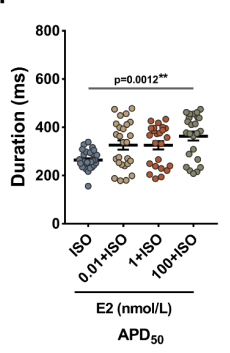

E

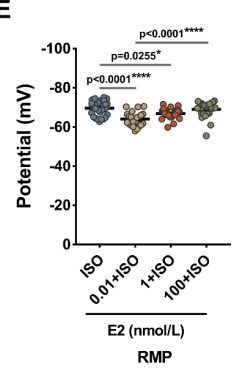

I

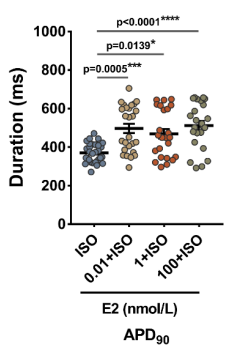

Supplemental Figure 5 E2 improves ISO induced abnormal action potential parameters of hiPSC-CMs. (AC) Typical APs and slope curve of hiPSC-CMs. (D-I) Values for APA, RMP, d $V / \mathrm{d} t \max , \mathrm{APD}_{10}, \mathrm{APD}_{50}$ and $\mathrm{APD}_{90} . \mathrm{n}=22-34$ cells, from $4-5$ petri dishes. ${ }^{*} p<0.05,{ }^{* *} p<0.01,{ }^{* * *} p<0.001,{ }^{* * * *} p<0.0001$. 

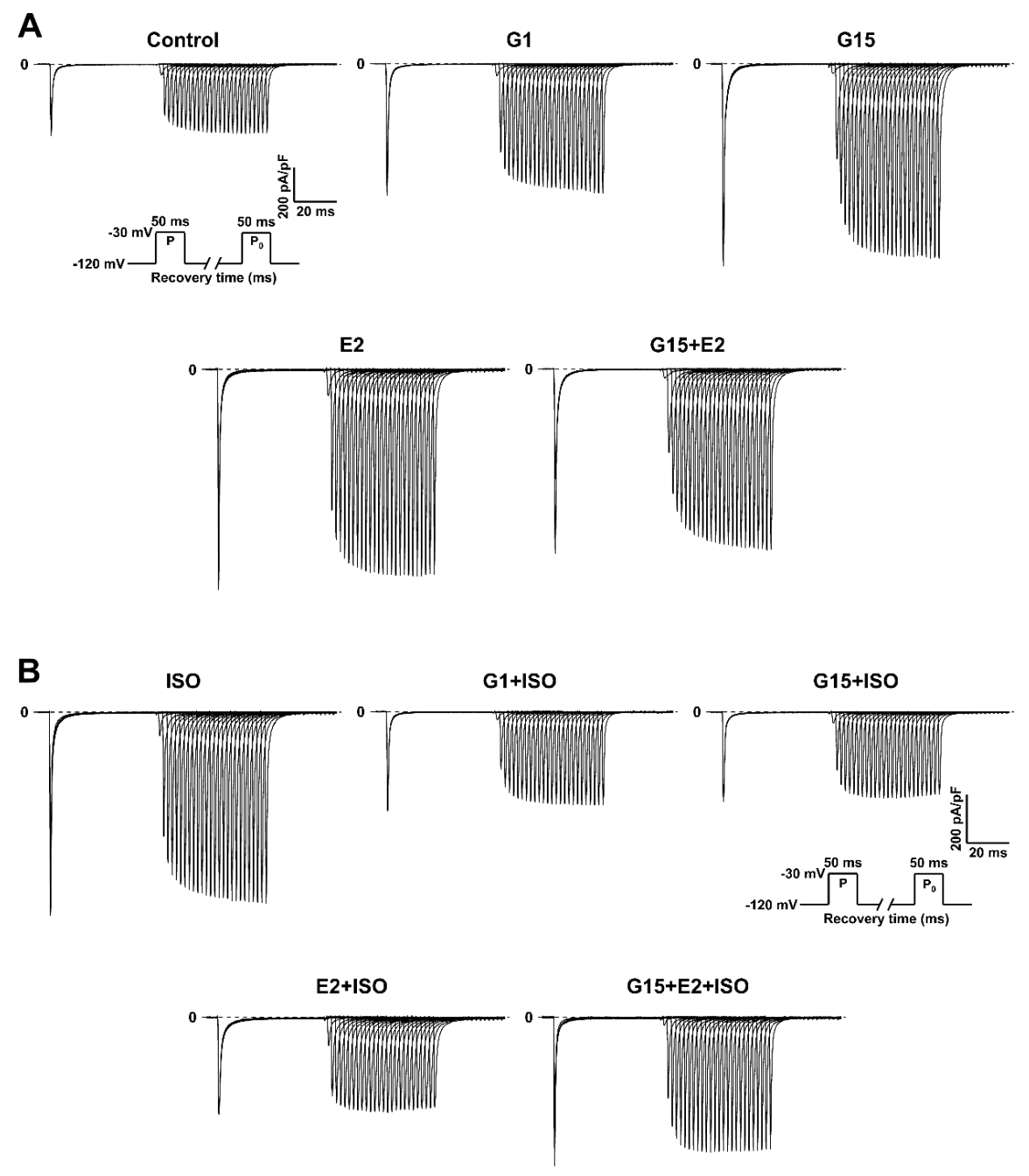

Supplemental Figure 6 Effects of GPER on the time constant of recovery from inactivation of sodium current in hiPSC-CMs. (A and B) Representative traces were showed in each group. Insets: voltage clamp protocols. 
A

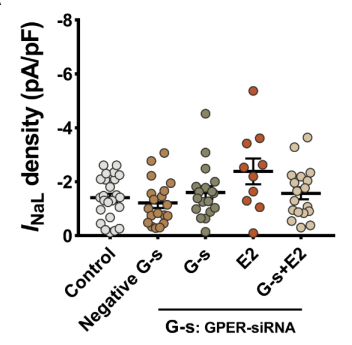

B

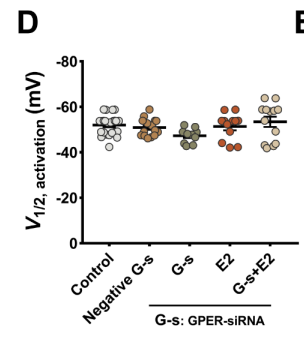

E

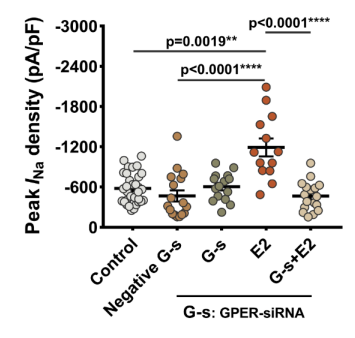

C

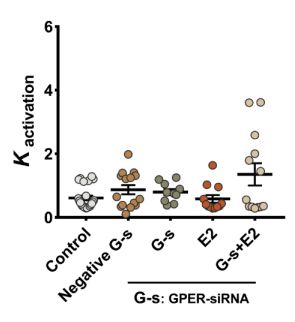

G

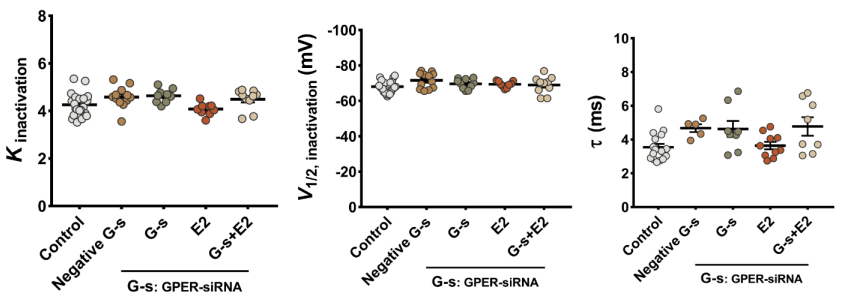

H

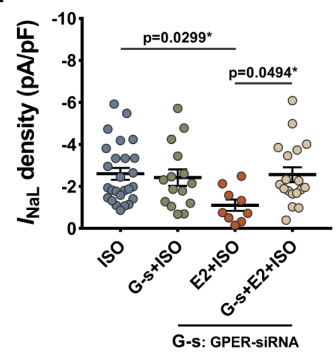

I

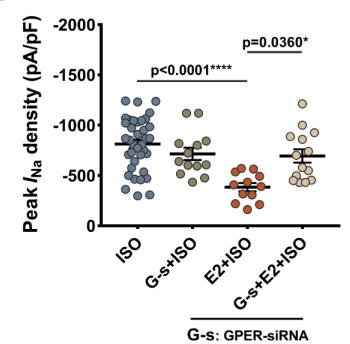

J

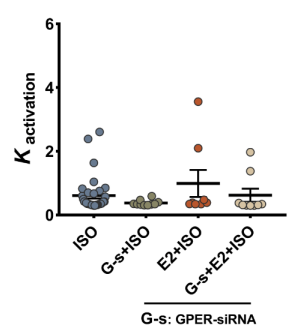

N

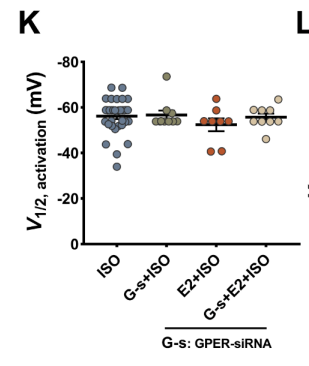

L

M
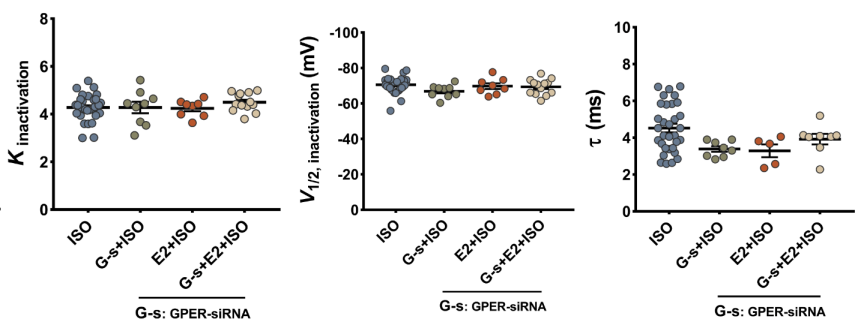

Supplemental Figure 7 Silence of GPER cancels the effect of estrogen on regulating peak $I \mathrm{Na}$ and $I \mathrm{NaL}$. (A) Average $I$ NaLdensity. $\mathrm{n}=10-25$ cells, from 4-9 Petri dishes. (B) Peak $I$ Na density. $\mathrm{n}=13-32$ cells, from 4-11 Petri dishes. (C-F) Values for $k$ and $\mathrm{V}_{1 / 2}$ of (in) activation. $\mathrm{n}=9-26$ cells, from 4-11 Petri dishes. $(\mathrm{G}) \tau$ value of recovery from inactivation. $\mathrm{n}=5-17$ cells, from 4-10 Petri dishes. $(\mathrm{H})$ Average $I$ NaL density. $\mathrm{n}=9-26$ cells, from 4-10 Petri dishes. (I) Peak $I$ Na density. $\mathrm{n}=12-37$ cells, from 4-11 Petri dishes. (J-M) Values for $k$ and $\mathrm{V}_{1 / 2}$ of (in) activation. $\mathrm{n}=8-34$ cells, from 4-11 Petri dishes. (N) $\tau$ value of recovery from inactivation. $\mathrm{n}=5$-32 cells, from 5-11 Petri dishes. One-way ANOVA was used. Kruskal-Wallis test was used selectively in the case of uneven variance. ${ }^{*} P<0.05,{ }^{* *} P<0.01,{ }^{* * * *} P<0.0001$. GPER, G protein coupled estrogen receptor. Insets: voltage clamp protocols. 

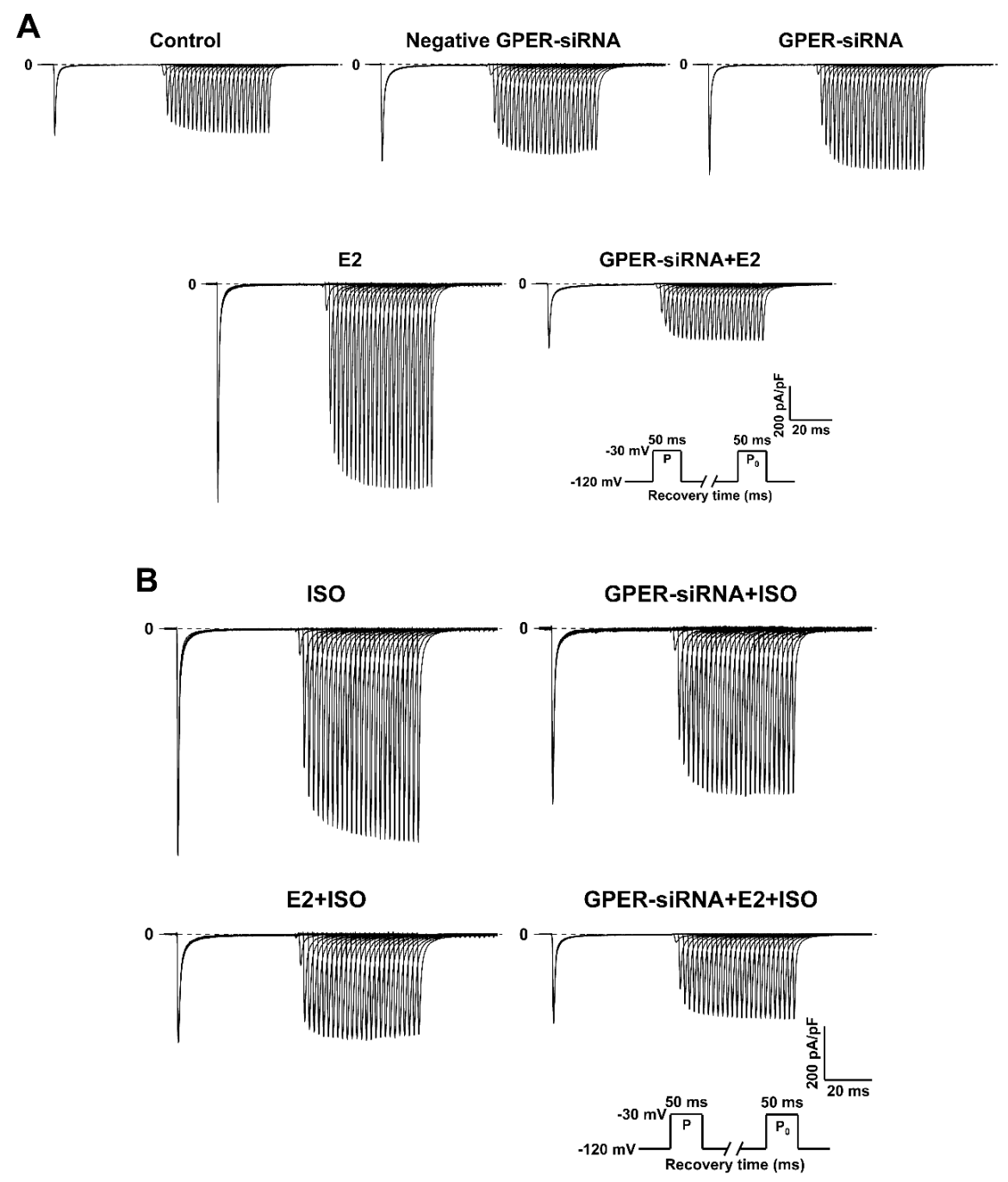

Supplemental Figure 8 Effects of silenced GPER on the time constant of recovery from inactivation of sodium current in hiPSC-CMs. (A and B) Representative traces were showed in each group. Insets: voltage clamp protocols.

Supplemental Table 1: Details in values of statistical analysis and the number of cells for average $I$ NaL density.

\begin{tabular}{llll}
\hline Group & Mean \pm S.E.M & $\mathrm{n}$ (cells) & Petri dishes \\
\hline Control & $-1.409 \pm 0.1558$ & 25 & 9 \\
$0.01 \mathrm{nmol} / \mathrm{L} \mathrm{E2}$ & $-2.299 \pm 0.4903$ & 8 & 3 \\
$1 \mathrm{nmol} / \mathrm{L} \mathrm{E2}$ & $-2.386 \pm 0.4755$ & 10 & 4 \\
$100 \mathrm{nmol} / \mathrm{L} \mathrm{E2}$ & $-1.938 \pm 0.2423$ & 14 & 4 \\
$\mathrm{ISO}$ & $-2.600 \pm 0.2790$ & 26 & 10 \\
$0.01 \mathrm{nmol} / \mathrm{L} \mathrm{E} 2+\mathrm{ISO}$ & $-2.209 \pm 0.4861$ & 12 & 4 \\
$1 \mathrm{nmol} / \mathrm{L} \mathrm{E2+ISO}$ & $-1.108 \pm 0.2739$ & 9 & 6
\end{tabular}




\begin{tabular}{llll}
\hline Group & Mean \pm S.E.M & $\mathrm{n}$ (cells) & Petri dishes \\
\hline 100 nmol/L E2+ISO & $-1.290 \pm 0.2608$ & 9 & 4 \\
G1 & $-1.547 \pm 0.1888$ & 13 & 4 \\
G15 & $-1.287 \pm 0.2632$ & 12 & 4 \\
G15+E2 & $-2.426 \pm 0.3696$ & 20 & 4 \\
G1+ISO & $-1.201 \pm 0.1890$ & 16 & 4 \\
G15+ISO & $-2.284 \pm 0.3592$ & 10 & 4 \\
G15+E2+ISO & $-2.644 \pm 0.3551$ & 12 & 4 \\
Negative GPER-siRNA & $-1.218 \pm 0.1891$ & 19 & 5 \\
GPER-siRNA & $-1.600 \pm 0.2371$ & 18 & 4 \\
GPER-siRNA+E2 & $-1.572 \pm 0.2237$ & 18 & 5 \\
GPER-siRNA+ISO & $-2.417 \pm 0.3920$ & 15 & 4 \\
GPER-siRNA+E2+ISO & $-2.559 \pm 0.3509$ & 18 & 4 \\
\hline
\end{tabular}

$I_{\mathrm{NaL}}$, late sodium current; E2, $\beta$-Estradiol; ISO, isoproterenol; G1, G protein-coupled estrogen receptor agonists; G15, G protein-coupled estrogen receptor antagonists; GPER, G protein-coupled estrogen receptor.

Supplemental Table 2 : Details in values of statistical analysis and the number of cells for peak $I$ Na.

\begin{tabular}{llll}
\hline Group & Mean \pm S.E.M & $\mathrm{n}$ (cells) & Petri dishes \\
\hline Control & $-577.7 \pm 40.21$ & 32 & 11 \\
$0.01 \mathrm{nmol} / \mathrm{L}$ E2 & $-1086 \pm 119.4$ & 10 & 4 \\
$1 \mathrm{nmol} / \mathrm{L}$ E2 & $-1191 \pm 133.8$ & 13 & 4 \\
$100 \mathrm{nmol} / \mathrm{L}$ E2 & $-865.3 \pm 82.75$ & 14 & 4 \\
$\mathrm{ISO}$ & $-811.8 \pm 43.41$ & 37 & 11 \\
$0.01 \mathrm{nmol} / \mathrm{L}$ E2+ISO & $-793.5 \pm 74.50$ & 12 & 4 \\
$1 \mathrm{nmol} / \mathrm{L}$ E2+ISO & $-384.2 \pm 41.11$ & 12 & 6 \\
$100 \mathrm{nmol} / \mathrm{L} \mathrm{E2+ISO}$ & $-588.0 \pm 69.50$ & 13 & 4 \\
G1 & $-885.8 \pm 75.24$ & 9 & 4 \\
G15 & $-651.3 \pm 57.12$ & 17 & 4 \\
G15+E2 & $-676.6 \pm 74.12$ & 21 & 4 \\
G1+ISO & $-536.5 \pm 44.83$ & 16 & 4 \\
G15+ISO & $-793.8 \pm 103.4$ & 13 & 4 \\
G15+E2+ISO & $-812.7 \pm 56.97$ & 19 & 4 \\
Negative GPER-siRNA & $-466.3 \pm 82.09$ & 17 & 5 \\
GPER-siRNA & $-603.8 \pm 54.23$ & 15 & 4 \\
GPER-siRNA+E2 & $-462.6 \pm 53.29$ & 17 & 5 \\
GPER-siRNA+ISO & $-714.5 \pm 61.06$ & 13 & 4 \\
GPER-siRNA+E2+ISO & $-694.2 \pm 66.75$ & 14 & 4 \\
\hline
\end{tabular}

$I_{\mathrm{Na}}$, sodium current; E2, $\beta$-Estradiol; ISO, isoproterenol; G1, G protein-coupled estrogen receptor agonists; G15, G protein-coupled estrogen receptor antagonists; GPER, G protein-coupled estrogen receptor.

Supplemental Table 3: Details in values of statistical analysis and the number of cells for $k$ of activation. 


\begin{tabular}{llll}
\hline Group & Mean \pm S.E.M & $\mathrm{n}$ (cells) & Petri dishes \\
\hline Control & $0.6123 \pm 0.07342$ & 24 & 10 \\
$0.01 \mathrm{nmol} / \mathrm{L}$ E2 & $0.4784 \pm 0.03594$ & 8 & 4 \\
$1 \mathrm{nmol} / \mathrm{L} \mathrm{E2}$ & $0.5839 \pm 0.1201$ & 12 & 4 \\
$100 \mathrm{nmol} / \mathrm{L}$ E2 & $0.5838 \pm 0.1079$ & 12 & 4 \\
$\mathrm{ISO}$ & $0.6146 \pm 0.09461$ & 34 & 11 \\
$0.01 \mathrm{nmol} / \mathrm{L}$ E2+ISO & $1.018 \pm 0.3049$ & 9 & 4 \\
$1 \mathrm{nmol} / \mathrm{L}$ E2+ISO & $0.9925 \pm 0.4239$ & 8 & 5 \\
$100 \mathrm{nmol} / \mathrm{L}$ E2+ISO & $1.217 \pm 0.3018$ & 10 & 4 \\
G1 & $0.5483 \pm 0.1588$ & 8 & 4 \\
G15 & $0.6775 \pm 0.09204$ & 15 & 4 \\
G15+E2 & $0.5658 \pm 0.08662$ & 15 & 4 \\
G1+ISO & $0.4632 \pm 0.1084$ & 13 & 4 \\
G15+ISO & $0.5109 \pm 0.08299$ & 12 & 4 \\
G15+E2+ISO & $0.6402 \pm 0.1313$ & 18 & 4 \\
Negative GPER-siRNA & $0.8718 \pm 0.1446$ & 15 & 5 \\
GPER-siRNA & $0.7959 \pm 0.09635$ & 10 & 4 \\
GPER-siRNA+E2 & $1.353 \pm 0.3517$ & 13 & 5 \\
GPER-siRNA+ISO & $0.3773 \pm 0.02849$ & 10 & 4 \\
GPER-siRNA+E2+ISO & $0.6217 \pm 0.2054$ & 9 & 4 \\
\hline
\end{tabular}

$k$ of activation, slope factor of activation; E2, $\beta$-Estradiol; ISO, isoproterenol; G1, G protein-coupled estrogen receptor agonists; G15, G protein-coupled estrogen receptor antagonists; GPER, G protein-coupled estrogen receptor.

Supplemental Table 4 : Details in values of statistical analysis and the number of cells for $\mathrm{V}_{1 / 2}$ of activation.

\begin{tabular}{llll}
\hline Group & Mean \pm S.E.M & $\mathrm{n}$ (cells) & Petri dishes \\
\hline Control & $-51.95 \pm 0.9018$ & 24 & 10 \\
$0.01 \mathrm{nmol} / \mathrm{L}$ E2 & $-52.63 \pm 2.250$ & 8 & 4 \\
$1 \mathrm{nmol} / \mathrm{L}$ E2 & $-51.37 \pm 1.660$ & 12 & 4 \\
$100 \mathrm{nmol} / \mathrm{L}$ E2 & $-54.44 \pm 1.769$ & 12 & 4 \\
$\mathrm{ISO}$ & $-56.16 \pm 1.297$ & 34 & 11 \\
$0.01 \mathrm{nmol} / \mathrm{L}$ E2+ISO & $-51.65 \pm 1.385$ & 9 & 4 \\
$1 \mathrm{nmol} / \mathrm{L}$ E2+ISO & $-52.42 \pm 2.842$ & 8 & 5 \\
$100 \mathrm{nmol} / \mathrm{L}$ E2+ISO & $-53.00 \pm 2.709$ & 10 & 4 \\
G1 & $-54.60 \pm 2.306$ & 8 & 4 \\
G15 & $-51.14 \pm 1.310$ & 15 & 4 \\
G15+E2 & $-49.89 \pm 1.385$ & 15 & 4 \\
G1+ISO & $-56.98 \pm 1.607$ & 13 & 4 \\
G15+ISO & $-57.33 \pm 1.956$ & 12 & 4 \\
G15+E2+ISO & $-55.11 \pm 1.302$ & 18 & 4 \\
Negative GPER-siRNA & $-50.91 \pm 0.9862$ & 15 & 5 \\
GPER-siRNA & $-47.32 \pm 1.015$ & 10 & 4 \\
GPER-siRNA+E2 & $-53.44 \pm 2.278$ & 13 & 5 \\
GPER-siRNA+ISO & $-56.68 \pm 1.960$ & 10 & 4 \\
GPER-siRNA+E2+ISO & $-55.68 \pm 1.644$ & 9 & 4 \\
\hline
\end{tabular}


$\mathrm{V}_{1 / 2}$ of activation, half-voltage of activation; E2, $\beta$-Estradiol; ISO, isoproterenol; G1, G protein-coupled estrogen receptor agonists; G15, G protein-coupled estrogen receptor antagonists; GPER, G protein-coupled estrogen receptor.

Supplemental Table 5: Details in values of statistical analysis and the number of cells for $k$ of inactivation.

\begin{tabular}{llll}
\hline Group & Mean \pm S.E.M & $\mathrm{n}$ (cells) & Petri dishes \\
\hline Control & $4.255 \pm 0.08697$ & 26 & 11 \\
$0.01 \mathrm{nmol} / \mathrm{L}$ E2 & $4.123 \pm 0.04982$ & 9 & 4 \\
$1 \mathrm{nmol} / \mathrm{L} \mathrm{E2}$ & $4.074 \pm 0.08570$ & 9 & 4 \\
$100 \mathrm{nmol} / \mathrm{L}$ E2 & $4.174 \pm 0.08291$ & 13 & 4 \\
ISO & $4.278 \pm 0.09169$ & 33 & 11 \\
$0.01 \mathrm{nmol} / \mathrm{L} \mathrm{E2+ISO}$ & $4.150 \pm 0.1370$ & 9 & 4 \\
$1 \mathrm{nmol} / \mathrm{L}$ E2+ISO & $4.243 \pm 0.1257$ & 8 & 5 \\
100 nmol/L E2+ISO & $4.106 \pm 0.08571$ & 12 & 4 \\
G1 & $4.070 \pm 0.09689$ & 9 & 4 \\
G15 & $4.225 \pm 0.09732$ & 14 & 4 \\
G15+E2 & $4.301 \pm 0.1188$ & 17 & 4 \\
G1+ISO & $4.195 \pm 0.1151$ & 15 & 4 \\
G15+ISO & $4.177 \pm 0.1031$ & 13 & 4 \\
G15+E2+ISO & $4.302 \pm 0.1036$ & 17 & 4 \\
Negative GPER-siRNA & $4.583 \pm 0.1292$ & 12 & 5 \\
GPER-siRNA & $4.638 \pm 0.08516$ & 10 & 4 \\
GPER-siRNA+E2 & $4.490 \pm 0.1358$ & 10 & 4 \\
GPER-siRNA+ISO & $4.275 \pm 0.2412$ & 9 & 4 \\
GPER-siRNA+E2+ISO & $4.499 \pm 0.1053$ & 13 & 4 \\
\hline
\end{tabular}

$k$ of inactivation, slope factor of inactivation; E2, $\beta$-Estradiol; ISO, isoproterenol; G1, G protein-coupled estrogen receptor agonists; G15, G protein-coupled estrogen receptor antagonists; GPER, G protein-coupled estrogen receptor.

Supplemental Table 6 : Details in values of statistical analysis and the number of cells for $\mathrm{V}_{1 / 2}$ of inactivation.

\begin{tabular}{llll}
\hline Group & Mean \pm S.E.M & $\mathrm{n}$ (cells) & Petri dishes \\
\hline Control & $-68.06 \pm 0.6328$ & 26 & 11 \\
$0.01 \mathrm{nmol} / \mathrm{L} \mathrm{E2}$ & $-69.75 \pm 0.9422$ & 9 & 4 \\
$1 \mathrm{nmol} / \mathrm{L} \mathrm{E2}$ & $-69.36 \pm 0.6028$ & 9 & 4 \\
$100 \mathrm{nmol} / \mathrm{L}$ E2 & $-66.86 \pm 0.9896$ & 13 & 4 \\
ISO & $-70.54 \pm 0.8001$ & 33 & 11 \\
$0.01 \mathrm{nmol} / \mathrm{L} \mathrm{E2+ISO}$ & $-67.75 \pm 1.809$ & 9 & 4 \\
$1 \mathrm{nmol} / \mathrm{L}$ E2+ISO & $-69.77 \pm 1.604$ & 8 & 5 \\
$100 \mathrm{nmol} / \mathrm{L} \mathrm{E2+ISO}$ & $-69.41 \pm 1.444$ & 12 & 4 \\
G1 & $-70.03 \pm 0.9939$ & 9 & 4 \\
G15 & $-69.32 \pm 0.7582$ & 14 & 4 \\
G15+E2 & $-70.11 \pm 0.9584$ & 17 & 4
\end{tabular}




\begin{tabular}{llll}
\hline Group & Mean \pm S.E.M & $\mathrm{n}$ (cells) & Petri dishes \\
\hline G1+ISO & $-68.95 \pm 0.7643$ & 15 & 4 \\
G15+ISO & $-69.87 \pm 0.7944$ & 13 & 4 \\
G15+E2+ISO & $-71.15 \pm 0.7586$ & 17 & 4 \\
Negative GPER-siRNA & $-71.58 \pm 1.278$ & 12 & 5 \\
GPER-siRNA & $-69.61 \pm 0.8926$ & 10 & 4 \\
GPER-siRNA+E2 & $-68.98 \pm 1.557$ & 10 & 4 \\
GPER-siRNA+ISO & $-66.90 \pm 1.183$ & 9 & 4 \\
GPER-siRNA+E2+ISO & $-69.36 \pm 1.231$ & 13 & 4 \\
\hline
\end{tabular}

$\mathrm{V}_{1 / 2}$ of inactivation, half-voltage of inactivation; E2, $\beta$-Estradiol; ISO, isoproterenol; G1, G protein-coupled estrogen receptor agonists; G15, G protein-coupled estrogen receptor antagonists; GPER, G protein-coupled estrogen receptor.

Supplemental Table 7 : Details in values of statistical analysis and the number of cells for $\tau$ of recovery from inactivation.

\begin{tabular}{llll}
\hline Group & Mean \pm S.E.M & $\mathrm{n}$ (cells) & Petri dishes \\
\hline Control & $3.544 \pm 0.2019$ & 17 & 10 \\
$0.01 \mathrm{nmol} / \mathrm{L}$ E2 & $3.594 \pm 0.1980$ & 8 & 4 \\
$1 \mathrm{nmol} / \mathrm{L}$ E2 & $3.641 \pm 0.2212$ & 10 & 4 \\
$100 \mathrm{nmol} / \mathrm{L}$ E2 & $3.350 \pm 0.4407$ & 11 & 4 \\
ISO & $4.522 \pm 0.2312$ & 32 & 11 \\
$0.01 \mathrm{nmol} / \mathrm{L}$ E2+ISO & $4.518 \pm 0.6411$ & 8 & 3 \\
$1 \mathrm{nmol} / \mathrm{L}$ E2+ISO & $3.291 \pm 0.3461$ & 5 & 4 \\
$100 \mathrm{nmol} / \mathrm{L}$ E2+ISO & $4.553 \pm 0.6519$ & 8 & 4 \\
G1 & $5.207 \pm 0.7400$ & 7 & 3 \\
G15 & $4.973 \pm 0.2979$ & 16 & 4 \\
G15+E2 & $4.717 \pm 0.4154$ & 15 & 4 \\
G1+ISO & $4.147 \pm 0.2508$ & 12 & 4 \\
G15+ISO & $4.116 \pm 0.3768$ & 11 & 4 \\
G15+E2+ISO & $4.991 \pm 0.2917$ & 14 & 4 \\
Negative GPER-siRNA & $4.676 \pm 0.2341$ & 5 & 4 \\
GPER-siRNA & $4.633 \pm 0.4730$ & 8 & 4 \\
GPER-siRNA+E2 & $4.779 \pm 0.5478$ & 8 & 4 \\
GPER-siRNA+ISO & $3.386 \pm 0.1518$ & 8 & 4 \\
GPER-siRNA+E2+ISO & $3.924 \pm 0.2886$ & 8 & 4 \\
\hline
\end{tabular}

$\tau$, time constant of recovery from inactivation; E2, $\beta$-Estradiol; ISO, isoproterenol; G1, G protein-coupled estrogen receptor agonists; G15, G protein-coupled estrogen receptor antagonists; GPER, G protein-coupled estrogen receptor.

Supplemental Table 8 : Details in values of statistical analysis and the number of cells for parameters of action potential. 


\begin{tabular}{llllll}
\hline Group & $\mathrm{APA}(\mathrm{mV})$ & $\mathrm{RMP}(\mathrm{mV})$ & $\mathrm{d} V / \mathrm{d} t_{\max }(\mathrm{mV} / \mathrm{ms})$ & $\mathrm{APD}_{10}(\mathrm{~ms})$ & $\mathrm{APD}_{50}$ \\
\hline Control $(\mathrm{n}=20-25$ cells $)$ & $113.8 \pm 0.9386$ & $-69.83 \pm 0.4187$ & $27.70 \pm 0.6309$ & $117.3 \pm 4.879$ & $302.6 \pm$ \\
$0.01 \mathrm{nmol} / \mathrm{L}$ E2 $(\mathrm{n}=31-34$ cells $)$ & $110.1 \pm 1.133$ & $-68.17 \pm 0.7299$ & $24.02 \pm 0.8293$ & $127.2 \pm 8.815$ & $326.6 \pm$ \\
$1 \mathrm{nmol} / \mathrm{L} \mathrm{E2} \mathrm{(n=19-21} \mathrm{cells)}$ & $107.9 \pm 1.021$ & $-67.57 \pm 1.012$ & $20.35 \pm 0.9915$ & $143.2 \pm 9.363$ & $376.6 \pm$ \\
$100 \mathrm{nmol} / \mathrm{L}$ E2 (n=35-40 cells) & $108.4 \pm 0.9901$ & $-66.57 \pm 0.7071$ & $23.41 \pm 0.7410$ & $130.8 \pm 5.379$ & $341.6 \pm$ \\
ISO (n=29-34 cells) & $109.4 \pm 1.482$ & $-69.64 \pm 0.6279$ & $21.74 \pm 0.7582$ & $97.59 \pm 5.575$ & $264.5 \pm$ \\
$0.01 \mathrm{nmol} / \mathrm{L}$ E2+ISO (n=22-28 cells) & $106.9 \pm 1.189$ & $-64.09 \pm 0.7868$ & $19.00 \pm 1.284$ & $115.1 \pm 6.775$ & $326.4 \pm$ \\
$1 \mathrm{nmol} / \mathrm{L}$ E2+ISO (n=24-27 cells) & $109.7 \pm 1.230$ & $-66.91 \pm 0.5038$ & $23.50 \pm 0.9637$ & $109.8 \pm 5.416$ & $325.6 \pm$ \\
$100 \mathrm{nmol} / \mathrm{L} \mathrm{E2+ISO} \mathrm{(n=24-26} \mathrm{cells)}$ & $113.8 \pm 1.434$ & $-69.04 \pm 0.7847$ & $21.82 \pm 0.9924$ & $118.5 \pm 5.812$ & $363.7 \pm$ \\
\hline
\end{tabular}

APA, action potential amplitude; RMP, resting membrane potential; $\mathrm{d} V / \mathrm{d} t$ max , maximal action potential upstroke velocity; $\mathrm{APD}_{10}$, action potential duration at $10 \%$ repolarization; $\mathrm{APD}_{50}$, action potential duration at $50 \%$ repolarization; $\mathrm{APD}_{90}$, action potential duration at $90 \%$ repolarization. E2, $\beta$-Estradiol; ISO, isoproterenol. Control, $0.01 \mathrm{nmol} / \mathrm{L} \mathrm{E} 2,1 \mathrm{nmol} / \mathrm{L} \mathrm{E} 2,100 \mathrm{nmol} / \mathrm{L} \mathrm{E2}$, ISO, $0.01 \mathrm{nmol} / \mathrm{L} \mathrm{E2+ISO,} 1 \mathrm{nmol} / \mathrm{L}$ $\mathrm{E} 2+\mathrm{ISO}$ and $100 \mathrm{nmol} / \mathrm{L} \mathrm{E2+ISO} \mathrm{group} \mathrm{originated} \mathrm{in} \mathrm{4,} \mathrm{5,} \mathrm{4,} \mathrm{6,} \mathrm{4,} \mathrm{5,} \mathrm{4,} 4$ Petri dishes respectively. 